\title{
Toll-like receptor 4 signaling in trigeminal ganglion neurons contributes tongue-referred pain associated with tooth pulp inflammation
}

Kinuyo Ohara ${ }^{1}$, Kohei Shimizu ${ }^{1,2^{*}}$, Shingo Matsuura ${ }^{1}$, Bunnai Ogiso ${ }^{1,2}$, Daisuke Omagari ${ }^{3}$, Masatake Asano ${ }^{3,4}$, Yoshiyuki Tsuboi ${ }^{5,6}$, Masamichi Shinoda ${ }^{5,6}$ and Koichi Iwata ${ }^{5,6,7}$

\begin{abstract}
Background: The purpose of the present study is to evaluate the mechanisms underlying tongue-referred pain associated with tooth pulp inflammation.

Method: Using mechanical and temperature stimulation following dental surgery, we have demonstrated that dental inflammation and hyperalgesia correlates with increased immunohistochemical staining of neurons for TLR4 and HSP70.

Results: Mechanical or heat hyperalgesia significantly enhanced in the ipsilateral tongue at 1 to 9 days after complete Freund's adjuvant (CFA) application to the left lower molar tooth pulp compared with that of sham-treated or vehicle-applied rats. The number of fluorogold (FG)-labeled TLR4-immunoreactive (IR) cells was significantly larger in CFA-applied rats compared with sham-treated or vehicle-applied rats to the molar tooth. The number of heat shock protein (Hsp) 70-IR neurons in trigeminal ganglion (TG) was significantly increased on day 3 after CFA application compared with sham-treated or vehicle-applied rats to the molar tooth. About 9.2\% of TG neurons were labeled with Dil applied to the molar tooth and FG injected into the tongue, and 15.4\% of TG neurons were labeled with FG injected into the tongue and Alexa-labeled Hsp70-IR applied to the tooth. Three days after Hsp70 or lipopolysaccharide (LPS) application to the tooth in naive rats, mechanical or heat hyperalgesia was significantly enhanced compared with that of saline-applied rats. Following successive LPS-RS, an antagonist of TLR4, administration to the TG for 3 days, the enhanced mechanical or heat hyperalgesia was significantly reversed compared with that of saline-injected rats. Noxious mechanical responses of TG neurons innervating the tongue were significantly higher in CFA-applied rats compare with sham rats to the tooth. Hsp70 mRNA levels of the tooth pulp and TG were not different between CFA-applied rats and sham rats.
\end{abstract}

Conclusions: The present findings indicate that Hsp70 transported from the tooth pulp to TG neurons or expressed in TG neurons is released from TG neurons innervating inflamed tooth pulp, and is taken by TG neurons innervating the tongue, suggesting that the Hsp70-TLR4 signaling in TG plays a pivotal role in tongue-referred pain associated with tooth pulp inflammation.

Keywords: Tooth-pulp inflammation, Tongue-referred pain, Trigeminal ganglion, Heat shock protein

\footnotetext{
* Correspondence: shimizu.kouhei01@nihon-u.ac.jp

${ }^{1}$ Department of Endodontics, Nihon University School of Dentistry, 1-8-13

Kandasurugadai, Chiyoda-ku, Tokyo 101-8310, Japan

${ }^{2}$ Divisions of Advanced Dental Treatment, Dental Research Center, Nihon

University School of Dentistry, Tokyo 101-8310, Japan

Full list of author information is available at the end of the article
} 


\section{Background}

It is well known that orofacial dysesthesia or referred pain sometimes occurs as a secondary hyperalgesia associated with tooth pulpal inflammation [1]. A number of previous studies have reported that the orofacial persistent pain following trigeminal nerve injury or orofacial inflammation is known to cause various motor as well as sensory disorders in the orofacial regions such as masticatory dysfunction and/or swallowing disorder [2]. It has also been reported that persistent pain is sometimes developed in oral structures following tooth pulp (TP) inflammation in human subjects [3]. Although sensitization of the peripheral nervous systems (PNS) following pulpal inflammation is thought to be involved in pathogenesis of the referred pain in oral structures, the peripheral mechanisms underlying orofacial-referred pain associated with TP inflammation remain unclear. Since the presence of orofacial dysesthesia and referred pain often lead to serious problems such as misdiagnosis and inappropriate treatment [4], it is necessary to clarify the mechanisms underlying orofacial dysesthesia and referred pain associated with TP inflammation.

Bacterial byproducts and various chemical mediators induced by peripheral infection or inflammation activate nociceptors in primary afferent neurons, and a barrage of action potentials are generated in primary afferent neurons which are sent to the central nervous system (CNS), resulting in the sensitization of nociceptive neurons in the PNS [5]. Substance P or calcitonin gene-related peptide is known to be released from trigeminal ganglion (TG) neurons following temporomandibular joint inflammation and these neuropeptides affect the excitability of adjacent TG neurons innervating the non-inflamed facial skin [6]. These findings strongly suggest that neuron-neuron interaction in the TG is involved in modulation of TG neurons innervating noninflamed oral structures following TP inflammation.

Toll-like receptors (TLRs) are known as transmembrane pattern-recognition receptors that initiate signals in response to diverse pathogen-associated molecular patterns (PAMPs). After tissue injury or cellular stress, TLRs detect endogenous ligands known as danger-associated molecular patterns (DAMPs) [7]. It is well known that TLRs in primary sensory neurons, such as dorsal root ganglion (DRG) and trigeminal ganglion (TG) neurons, are involved in the modulation of neuronal excitation, in particular, primary sensory neurons expressing TLR4 and TLR7 to sense exogenous PAMPs and endogenous DAMPs released after tissue injury or cellular stress [8]. These neuronal TLRs are thought to be involved in the development of pathological pain following peripheral inflammation.

Heat shock protein 70 (Hsp70) is well known as a specific ligand for TLRs and has been reported to be expressed in the brain [9] and heart [10], and is involved in pathological pain associated with tissue injury or inflammation
[11]. Previous studies have also reported that Hsp70 is expressed in the dental pulp following pulpal trauma or inflammation [12], therefore it is highly possible that Hsp70 is involved in the development of pathological pulpal pain and orofacial referred pain.

Together, we hypothesized that Hsp70 was expressed in the pulpal tissue or TG neurons after the TP inflammation and affected the excitability of TG neurons through TLR4 signaling, and played a crucial role in the development of tongue-referred pain. To test this hypothesis, we analyzed mechanical- and heat-evoked nocifensive reflex, TLR4 expression in TG after pulpal inflammation, primary afferent tracing using fluorogold (FG) and DiI applied into the tongue and lower first molar tooth (M1) to study if the single TG neuron innervates both pulp and tongue, Hsp70 expression in the TG after pulpal inflammation, fluorescent labeled-Hsp70 expression in TG neurons via axonal flow following labeled Hsp70 injection into the left M1TP, TG neuronal activity, RT-PCR analysis of Hsp70 in the inflamed tooth pulp and TG, and the effect of TLR4 agonist and antagonist on the nocifensive reflex.

\section{Methods}

\section{Animals}

This study was approved by the Animal Experimentation Committee at Nihon University. All surgery and animal care were conducted in accordance with the National Institutes of Health Guide for the Care and Use of Laboratory Animals and the guidelines for Institutional Animal Care, and the guidelines of the International Association for the Study of Pain [13]. Male Sprague-Dawley rats $(n=$ 177, Japan SLC, Shizuoka, Japan) weighing 250 to $350 \mathrm{~g}$ were used in this study. The animals were maintained in a temperature-controlled room $\left(23^{\circ} \mathrm{C}\right)$ with a $12 / 12$-h light/ dark cycle. Food and water were freely available.

\section{CFA application to the TP}

Rats were lightly anesthetized with $2 \%$ isoflurane (Mylan, Canonsburg, PA, USA) and then deeply anesthetized with an intraperitoneal (i.p.) application of sodium pentobarbital $(50 \mathrm{mg} / \mathrm{kg}$; Schering Plough, Whitehouse Station, NJ, USA). Then the rats were placed on a warm mat $\left(37^{\circ} \mathrm{C}\right)$ in the supine position to allow for the application of CFA (Sigma-Aldrich, St. Louis, MI, USA). A total of 50\% CFA (diluted in saline) or vehicle (isotonic saline) was applied to the M1 unilaterally. The rat's mouth was gently opened and the left M1TP was exposed by means of a low-speed dental drill with a round tungsten carbide bur under water cooling. A small piece of dental paper point (diameter, $0.15 \mathrm{~mm}$; length, $1.5 \mathrm{~mm}$ ) soaked with CFA or vehicle was applied to the exposed M1TP. Then the exposed pulp cavity was sealed with dental cement. 


\section{Head-withdrawal reflex threshold measurement}

The head-withdrawal reflex threshold (HWT) to mechanical and heat stimulation of the lateral edge of tongue (3 $\mathrm{mm}$ posterior from tip of tongue) was measured on day 3 after saline or CFA application to the tooth pulp, under light anesthesia with 2\% isoflurane (Mylan, Canonsburg, PA, USA) in oxygen. Bipolar enamel-coated stainless steel wire electrodes (Narishige, Tokyo, Japan) were placed in the splenius capitis muscle for electromyogram (EMG) recording of the reflex response (inter-electrode distance, 5 to $6 \mathrm{~mm}$ ).

Lower jaw was gently pulled with plastic strings and rat's mouth was kept open, and then mechanical stimulation ( 0 to $130 \mathrm{~g} ; 10 \mathrm{~g} / \mathrm{s}$; cutoff, $130 \mathrm{~g}$ ) was applied to the lateral edge of the tongue ipsilateral to the CFA or vehicle application by using forceps with flat tips $\left(4 \mathrm{~mm}^{2}\right.$; Panlab s.l., Barcelona, Spain) in lightly anesthetized rats ( $n=7$ in each group). The stimulus velocity was manually controlled consecutively from $0 \mathrm{~g}$ to threshold values at a speed of approximately $10 \mathrm{~g} / \mathrm{s}$. The threshold intensity for evoking EMG activity by mechanical stimulation of the tongue was defined as the mechanical HWT.

Heat stimulation $\left(35-60^{\circ} \mathrm{C} ; 1^{\circ} \mathrm{C} / \mathrm{s}\right.$; cutoff, $\left.60^{\circ} \mathrm{C}\right)$ was also applied to the lateral edge of the tongue ipsilateral to the CFA or vehicle application by using a contact heat probe ( $9 \mathrm{~mm}^{2}$; Intercross, Tokyo, Japan) in lightly anesthetized rats ( $n=7$ in each group). The threshold temperature for evoking EMG activity by heat stimulation to the tongue was defined as the heat HWT. The mechanical or heat stimulation was applied three times with 5-min intervals, and the mean value of the HWTs was calculated. The splenius capitis EMG was recorded following mechanical and heat stimulation of the tongue on days $1,3,5,7,9,11,14,21,28$ (days 21, 28 data not shown.). Time-course change in mean HWT value to mechanical or heat stimulation of the ipsilateral tongue was measured in CFA, vehicle, or sham rats. The baseline HWT value to mechanical or heat stimulation was measured before CFA or vehicle application, or sham treatment of the tooth.

\section{TLR4 immunohistochemistry in combination with FG tracer}

For TLR4 immunohistochemistry in combination with FG tracer into tongue, $5.0 \mu \mathrm{L}$ of $10 \%$ FG (Wako) dissolved in saline was applied into the lateral edge of the tongue of the rats anesthetized with $2 \%$ isoflurane. On day 3 after FG application, rats were lightly anesthetized with $2 \%$ isoflurane and then deeply anesthetized with sodium pentobarbital $(50 \mathrm{mg} / \mathrm{kg}$, i.p.) for the application of CFA or vehicle into the M1TP. On day 3 after CFA or vehicle application, rats were transcardially perfused with saline, followed by a fixative containing $4 \%$ paraformaldehyde in $0.1 \mathrm{M}$ phosphate buffer ( $\mathrm{pH}$ 7.4) under same anesthesia used for CFA or vehicle application ( $n=5$ in each group). TGs in the ipsilateral side to CFA, vehicle, or sham operation were dissected out after perfusion and post-fixed in 4\% PFA for 1 day at $4^{\circ} \mathrm{C}$. The specimens were then transferred to $20 \%$ sucrose $(\mathrm{w} / \mathrm{v})$ in distilled water for several days for cryopreservation, were then embedded in Tissue Tek (Sakura Finetek, Torrance, CA, USA), and stored until cryosectioning at $-20^{\circ} \mathrm{C}$. TG sections of $10 \mu \mathrm{m}$ were cut in the horizontal plane along the longitudinal axis. Every eighth section was thaw-mounted on MAS-GP microslide glass (Matunami, Osaka, Japan) and dried overnight at room temperature. Four sections were chosen from each TG in each rat and these were processed for TLR4 immunohistochemistry. Sections were incubated with rabbit anti-TLR4 polyclonal antibody (1:200; abcam) after dilution at a concentration of 1:800 in $0.01 \mathrm{M}$ PBS containing $4 \%$ normal goat serum (NGS) and $0.3 \%$ Triton X-100 (Sigma-Aldrich) on day 3 at $4^{\circ} \mathrm{C}$. After rinsing with $0.01 \mathrm{M}$ PBS, sections were incubated in Alexa Fluor 488 goat antirabbit IgG (1:200 in 0.01 M PBS; Invitrogen, Paisley, UK) for $2 \mathrm{~h}$ at room temperature. After rinsing with $0.01 \mathrm{M}$ PBS, sections were cover-slipped in mounting medium (Thermo Fisher Scientific, Fremont, CA, USA) and examined under a fluorescence microscope and analyzed using a BZ-9000 system (Keyence, Osaka, Japan). No specific labeling was observed in the absence of primary antibody. The number of TLR4-IR cells in TG in the V3 branch region were analyzed and counted in each rat (SensiveMeasure; Mitani, Fukui, Japan; $n=5$ in each group). In addition, the relative numbers of them were calculated by the following formula: $100 \times$ number of neurons for TLR4- or FG-IR cells/FG-IR cells.

\section{Hsp70 immunohistochemistry}

For Hsp70 immunohistochemistry, sections were incubated with rabbit anti-Hsp70 polyclonal antibody (1:200; abcam) after dilution at a concentration of 1:800 in $0.01 \mathrm{M}$ PBS containing 4\% NGS and 0.3\% Triton X-100 (SigmaAldrich) on day 3 at $4^{\circ} \mathrm{C}$. After rinsing with $0.01 \mathrm{M}$ PBS, sections were incubated in Alexa Fluor 488 goat antirabbit IgG (1:200 in 0.01 M PBS; Invitrogen, Paisley, UK) for $2 \mathrm{~h}$ at room temperature. After rinsing with $0.01 \mathrm{M}$ PBS, sections were cover-slipped in mounting medium (Thermo Fisher Scientific, Fremont, CA, USA) and examined under a fluorescence microscope and analyzed using a BZ-9000 system (Keyence, Osaka, Japan). No specific labeling was observed in the absence of primary antibody. The number of Hsp70-IR cells in TG in the V3 branch region were analyzed and counted in each rat (SensiveMeasure; Mitani, Fukui, Japan; $n=5$ in each group). In addition, the relative numbers of them were calculated by the following formula: $100 \times$ number of Hsp70-IR cells/all TG neurons. 


\section{Labeling of recombinant Hsp70}

The Alexa Fluor 594 labeling of Hsp70 (R\&D Systems) was performed with Alexa Fluor 594 microscale protein labeling kit (Molecular Probes). Briefly, $50 \mu \mathrm{g}$ of Hsp70 was mixed with $5.0 \mu \mathrm{L}$ of $1 \mathrm{M}$ sodium bicarbonate and pipetted thoroughly. The sample was further mixed with $2.6 \mu \mathrm{L}$ of Alexa Fluor 594 solution and incubated for $15 \mathrm{~min}$ at room temperature. The reaction mixture was applied to the spin column and unreacted dye was eliminated. The flowthrough was collected and the protein concentration was measured. The labeled protein was aliquoted and stored at $-80^{\circ} \mathrm{C}$ until use.

\section{FG, Dil, and recombinant Hsp70 application to the pulp}

For FG, DiI, and labeled recombinant Hsp70 application to the pulp, rats were deeply anesthetized with sodium pentobarbital $(50 \mathrm{mg} / \mathrm{kg}$, i.p.) and $5.0 \mu \mathrm{L}$ of $10 \%$ FG dissolved in saline was applied into the lateral edge of the tongue. On day 4 after FG application, DiI (Invitrogen) saturated in 100\% ethanol or labeled recombinant Hsp70 was also applied into M1 with paper point. Subsequently on day 3 , rats were deeply anesthetized and perfused, and then TG was removed and sectioned. FG- and/or recombinant Hsp70-labeled cells, or FG- and/or DiI-labeled cells were studied in TG under fluorescent microscopy, respectively.

\section{Hsp70 or LPS application to the M1TP}

Rats were anesthetized with sodium pentobarbital (50 mg/ kg, i.p.), the recombinant Hsp70 or LPS was applied to the left M1TP with the same procedures as CFA application to the M1TP. On day 3 after drug application HWT to mechanical and heat stimulation of the tongue was measured. Saline was also administered as vehicle control.

\section{LPS-RS administration into TG}

Rats were anesthetized, and a small hole (diameter, $1 \mathrm{~mm}$ ) was drilled in the skull above V1/V2 and V3 branch of TG. The guide cannula was extended into the hole $9 \mathrm{~mm}$ below the skull surface into TG and was fixed to the skull with three stainless-steel screws and dental resin, according to the method by Katagiri et al. [14]. CFA- rats were administered saline $(0.5 \mu \mathrm{L})$ or TLR4 antagonist LPS-RS $(0.1 \mathrm{mM} 0.5 \mu \mathrm{L} /$ day; Invivo Gen) dissolved in saline $(n=5$ in each group), and naive rats were administrated saline $(0.5 \mu \mathrm{L})$ or LPS-RS $(0.5 \mu \mathrm{L} /$ day; Invivo Gen) dissolved in saline ( $n=5$ in each group) once a day into TG for 3 successive days (day 0 through day 2) ( $\mathrm{n}=5$ in each group). HWTs were then measured on day 3 day under light anesthesia with $2 \%$ isoflurane in oxygen.

\section{Single neuron recording from TG neurons}

Three days after CFA application to the M1TP or sham treatment of the M1 teeth, rats were anesthetized with pentobarbital-Na (50 mg/kg, i.p.). Trachea and femoral vein were cannulated for artificial respiration and intravenous administration of drugs, and then rats were fixed in the stereotaxic flame. Brain tissue over the TG was removed and TG surface was exposed, and the enamelcoated tungsten electrodes were inserted into the TG and single neuronal activities were recorded. During the recording session, rats were immobilized with pancuronium bromide $(0.6 \mathrm{mg} / \mathrm{kg} / \mathrm{h}$, i.v. Schering-Plough, Darmstadt, Germany) and artificially ventilated, and end-tidal $\mathrm{CO}_{2}$ concentration and body temperature were maintained at 3.5 to $4.5 \%$ and at $37^{\circ} \mathrm{C}$ by a feedback-controlled heating blanket (Nihon koden, Tokyo, Japan). We used gentle brush and pressure stimuli as the search stimuli. When neuronal activity was obtained, brush, pressure, or noxious pinch (50 g) was applied to the receptive fields for $5 \mathrm{~s}$ with camel brush, brunt forceps, or small arterial clip (50 g), respectively. Spontaneous activity was recorded for $1 \mathrm{~min}$ before application of brush or pressure stimulus.

\section{Real-time PCR}

Total RNA was purified using an RNeasy mini kit (QIAGEN, Tokyo, Japan). One $\mu g$ of total RNA was subjected to first-strand cDNA synthesis with Superscript III reverse transcriptase (Life Technologies, Carlsbad, CA, USA), as previously described [15]. Real-time PCR was performed using LightCycler ${ }^{\circledR}$ Nano (Roche, Tokyo, Japan) with SYBR green (TaKaRa, Tokyo, Japan). The Hsp70 and GAPDH primers were purchased from TaKaRa.

\section{Statistical analysis}

Data were expressed as means \pm SEM. Statistical analyses were performed by student's $t$-test, or one-way ANOVA or two-way repeated-measures ANOVA followed by Bonferroni's multiple comparison tests where appropriate. A value of $P<0.05$ was considered as significant.

\section{Results}

\section{Nocifensive reflex to mechanical or heat stimulation of} the tongue

The HWT to mechanical or heat stimulation of the ipsilateral tongue significantly decreased at 1 to 9 days after CFA application to the M1TP compared with that of shamtreated or vehicle-applied rats (mechanical, $P<0.001$; heat, $P<0.001 ; n=7$ in each group) (Figure 1A: mechanical, B: heat). We also observed significant decrease in the headwithdrawal threshold to mechanical stimulation of the tongue in vehicle-applied rats at 1 to 9 days after vehicle application compared with sham rats $(P<0.001)$, and on days 3 and 7 heat head-withdrawal threshold was significantly lower in vehicle-applied rats compared to sham rats $(P<0.001)$. Sham-treated rats did not show any changes in HWT to mechanical or heat stimulation of the tongue. 

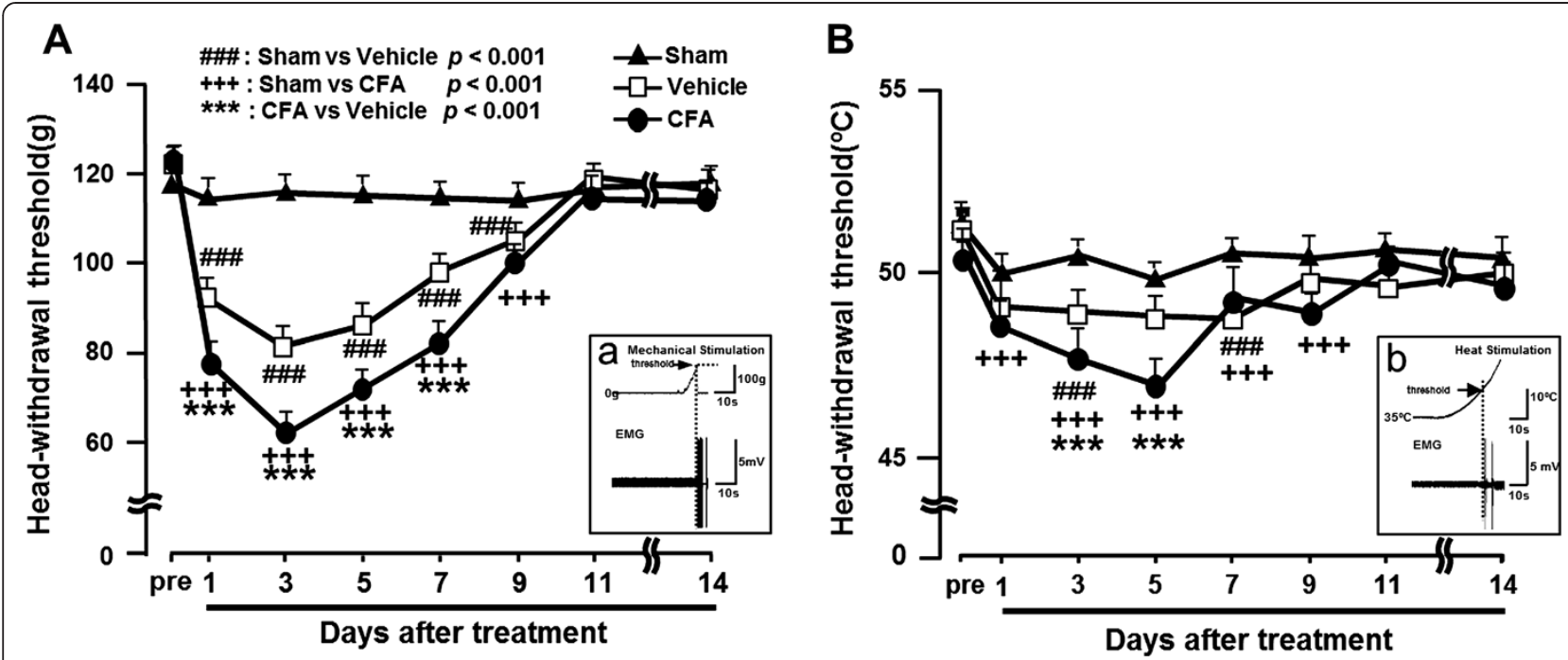

Figure 1 Tongue mechanical/thermal hypersensitivity following CFA application to the tooth pulp in lightly anesthetized rats. (A) Head-withdrawal threshold (HWT) to mechanical stimulation of the tongue. (B) HWT to heat stimulation of the tongue. Aa and Bb: EMG recordings from trapezius muscles during mechanical or heat stimulation of the tongue. \#\#\#: $P<0.001$ (sham vs. vehicle), $+++: P<0.001$ (sham vs. CFA), ***: $P<0.001$ (CFA vs. vehicle).

\section{TLR4 expression in TG neurons}

To study if TG cells innervating the tongue show TLR4-IR following CFA application to M1TP, FG was injected into the tongue and then CFA was applied to the M1TP. Following CFA application to the M1TP, many TG neurons showed TLR4-IR, and some of them were retrogradely labeled with FG injected into the tongue (Figure 2A). The number of FG-positive TLR4-IR TG neurons was counted in the V3 branch region (the area indicated by the arrow in Figure 2B). The mean relative number of FG-labeled TLR4IR cells was significantly increased in M1TP CFA-applied rats compared with M1TP sham and vehicle-applied rats ( $n=5$ in each group) (Figure 2C).

\section{Hsp70 expression following M1TP inflammation}

A small number of Hsp70-IR cells were observed in TG in sham- and vehicle- applied rats, whereas a larger number of Hsp70-IR cells were seen in the TG of CFA-applied rats (Figure 3A,B, and C). The mean relative number of Hsp70IR TG neurons was significantly increased on day 3 after CFA application into the M1TP compared with sham or vehicle-applied rats ( $n=5$ in each group) (Figure 2D).

\section{FG, Dil, and labeled recombinant Hsp70 tracing}

We also studied if the TG neurons were retrogradely labeled with both FG and DiI injected into the tongue and M1, respectively (Figure 3E,F,G, and H). About 9.2\% $(n=5)$ of TG neurons were labeled with FG and DiI (Figure $3 \mathrm{H}$ ), indicating that some TG neurons innervate both tongue and M1TP.

We further studied whether fluorescent-labeled recombinant Hsp70 applied to the M1TP was transported to TG neurons in rats with FG injection into the tongue (Figure 3I, J, K, and L). About 15.4\% ( $n=5)$ of FG-labeled TG neurons were labeled with recombinant Hsp70 (Figure 3L), indicating that fluorescent-labeled recombinant Hsp70 was transported from the M1 tooth pulp to TG neurons and released, and was taken by TG neurons innervating into the tongue.

\section{Effect of Hsp70 or LPS administration into TG on HWT}

Three days after recombinant Hsp70 application to the M1TP in naive rats, the HWTs to mechanical and heat stimulation of the tongue were significantly decreased compared with that of saline-applied rats $(P<0.001, n=$ 5 in each group) (Figure 4A and B).

We also observed significant reduction of the HWT to mechanical and heat stimulation of the tongue at 3 days after LPS application to the M1TP in sham rats compared with saline-applied rats $(P<0.001, n=5$ in each group) (Figure $4 \mathrm{C}$ and D).

\section{Effect of LPS-RS administration into TG on HWT}

Following successive LPS-RS administration $(0.5 \mu \mathrm{L} /$ day $)$ for 3 days into TG in M1 CFA-applied rats, the decreased HWTs to mechanical and heat stimulation of the tongue were significantly reversed compared with that of salineinjected rats $(P<0.001, n=5$ in each group) (Figure $4 \mathrm{E}$ and F).

\section{TG neuronal activity}

To test if TG neurons innervating the tongue increased their activities following CFA application to the M1TP, single neuronal activities innervating the tongue were 


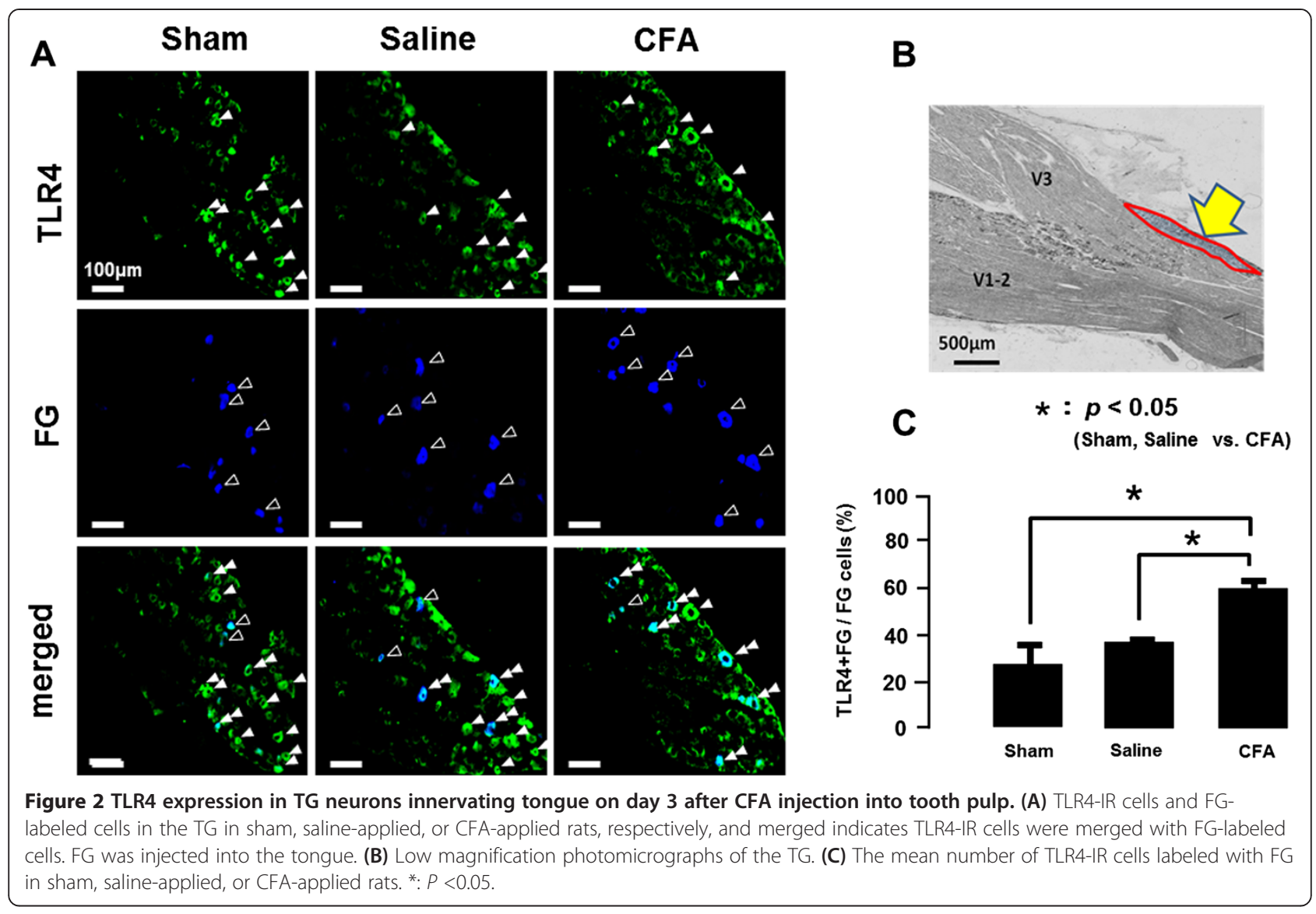

analyzed in the M1TP-inflamed or sham rats. A total of 26 TG nociceptive neurons innervating the tongue were classified as TG neurons with superficial receptive fields responding to brush (superficial RF neurons; sham, $n=9$; CFA, $n=10$ ) or deep receptive fields responding to brush (deep RF neurons; sham, $n=9$; CFA, $n=8$ ), respectively. No significant changes in spontaneous activities in superficial and deep RF neurons following CFA application to the M1TP (Figure 5A and B). On the other hand, superficial but deep RF neurons showed significant increase in firing frequency following noxious mechanical stimulation of the RFs in M1TP CFA-applied rats compared with sham rats (Figure $5 \mathrm{C}$ and $\mathrm{D}$ ).

\section{Hsp70 mRNA expression}

We also studied the change in Hsp70 mRNA level in the M1TP and TG following M1TP inflammation using RTPCR technique (Figure 6). No significant differences of Hsp70 mRNA levels in the M1TP and TG between CFAapplied rats and sham rats.

\section{Discussion}

This study has provided novel evidence suggesting a role of TLR4 mediating hyperexcitability of TG neurons innervating the non-inflamed tongue resulting in ectopic tongue pain associated with TP inflammation. To evaluate the mechanisms underlying ectopic tongue pain following TP inflammation, measurement of nocifensive reflex and immunohistochemical studies were conducted. We demonstrated that mechanical and heat HWT were significantly reduced, TLR4 and Hsp70 expression in the TG and the excitability of TG nociceptive neurons innervating the tongue were strongly enhanced, Alexa labeled-Hsp70 was transported from the M1TP to TG neurons, and there were some TG neurons innervating both M1TP and tongue. Furthermore, Hsp70 or LPS application to the M1TP caused the decrease in HWT, and LPS-RS to the TG attenuated the enhanced nocifensive reflex.

\section{TLR4 expression in TG neurons}

Previous studies have reported that TLRs expressed in the dorsal ganglion neurons are key molecules involved in modulation of neuronal excitability and respond to endogenous ligands following tissue injury or inflammation [16-18]. Increasing evidence indicates that TLRs and their associated signals contribute to pathological pain conditions, and blockade of TLR signaling has been shown to reduce hyperalgesia $[18,19]$. It has also been reported that CFA injection into the lower lip causes strong enhancement of the excitability of trigeminal ganglion (TG) neurons 


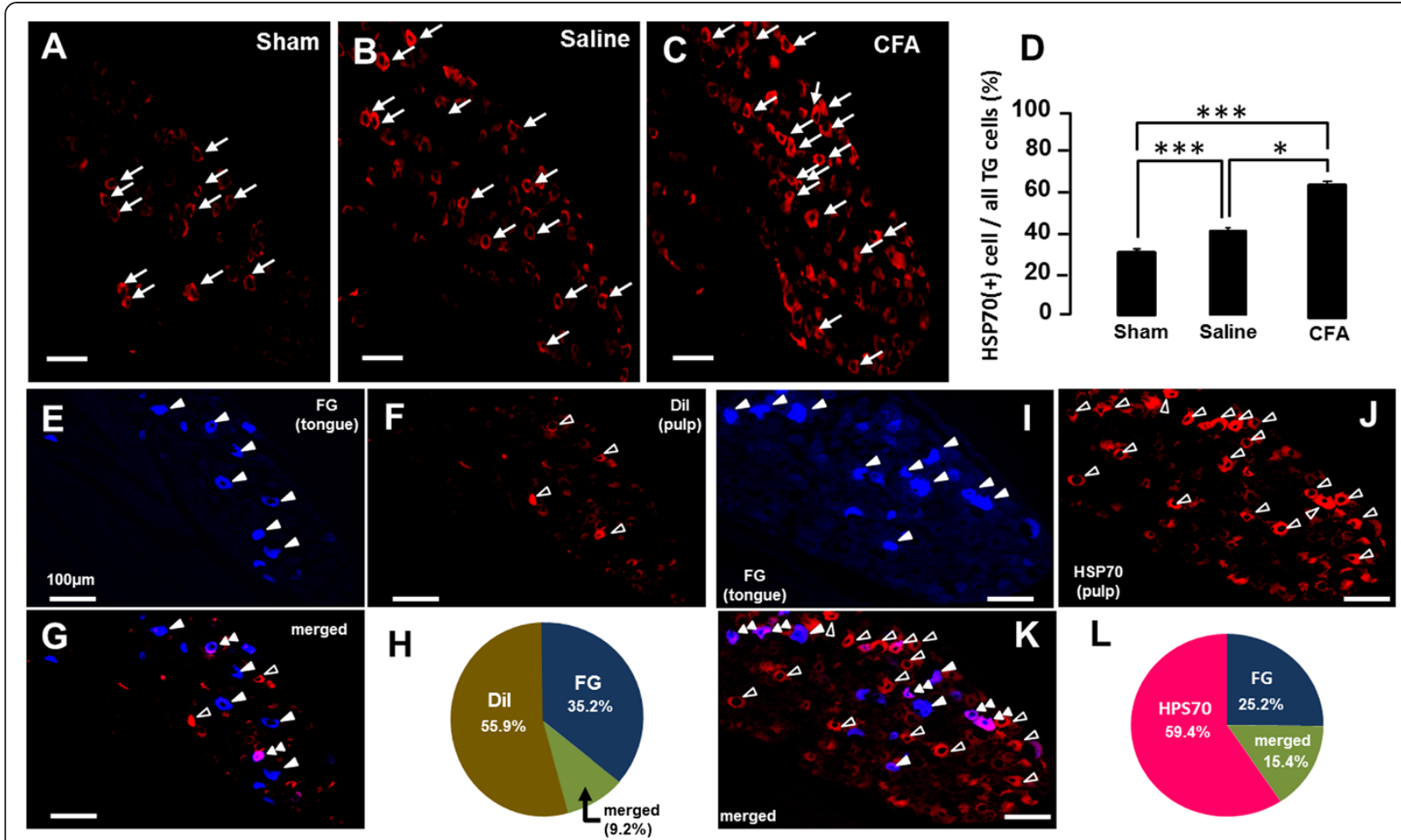

Figure 3 Hsp70 expression in the TG neurons in sham, saline-applied, or CFA-applied rats, and FG-, Dil-, or Hsp70-labeled cells in the TG. A, B, C: Hsp70-IR cells in the TG in sham (A), saline-applied (B), or CFA-applied rats (C). (D) The mean number of Hsp70-IR cells in the TG in sham, saline-applied or CFA-applied rats. E, F, G: FG- (E) and Dil-labeled cells (F) or Alexa-labeled FG-labeled cells merged with Dil-labeled cells (G) in the TG. (H): The pie graph indicates the percentage of Dil, FG, or merged cells in the TG. I, J, K: FG- (I) and Alexa-labeled Hsp70-labeled cells (J) or FG-labeled cells merged with HSP70-labeled cells (K) in the TG. (L) The pie graph indicates the percentage of FG-, Alexa-labeled Hsp70, or merged cells in the TG. Note that intra-pulpal Hsp70 was axonally transported to TG on day 3 after Alexa-labeled Hsp70 injection into the tooth pulp. *: $P<0.05,{ }^{* *}: P<0.001$.

innervating non-inflamed upper lip via neuron-neuron interactions [20-22]. We showed the significant enhancement of nocifensive reflex to mechanical and heat stimulation of the tongue following M1TP inflammation, and also observed significant increase in the number of TLR-IR TG neurons innervating the non-inflamed tongue compared with vehicle-applied rats. Together with previous data our results suggest that the TLR4 expressed in TG neurons is a key receptor for ectopic tongue pain associated with M1TP inflammation.

\section{TLR4 ligands in TG neurons}

LPS and Hsp70 are known as the specific ligands for the TLR4, and these molecules bind to the TLR4 expressed in neurons, resulting in the enhancement of neuronal excitability $[11,18]$. It is also well known that Hsps localize within the cells and bind to heat shock factors, and following an appropriate stress, accumulation of unfolded proteins leads to the dissociation of Hsps from heat shock factors, leaving Hsps free to bind target proteins [23]. On the other hand, LPS is a major cell wall component of Gram-negative bacteria and TLR4 exogenous ligand is recognized by TLR4. We observed strong expression of TLR4 and Hsp70 in TG neurons innervating non-inflamed tongue which is associated with M1TP inflammation. Hsp70 is one of the ligands expressed in inflamed tissues and acts as a ligand for TLR4 [8,23,24]. Following intraganglionic administration of Hsp70 or LPS caused mechanical and heat hyperalgesia in naive rats, and the enhanced mechanical and heat nocifensive reflexes were strongly attenuated by LPS-RS injection to the TG in the rats with M1TP inflammation. Furthermore, we observed significant enhancement of the excitability of TG nociceptive neurons innervating the tongue in M1TP inflamed rats compared with sham rats. These results suggest that Hsp70 expressed in TG neurons binds to TLR4 in TG neurons innervating the tongue and then increases the excitability of those TG neurons following M1TP inflammation.

Based on the previous results and present data, Hsp70 expression after tooth pulp inflammation may have two different functions in accordance with its location; Hsp70 in the tooth pulp exerts neuroprotective, cytoprotective, and housekeeping functions as a chaperone protein, whereas 

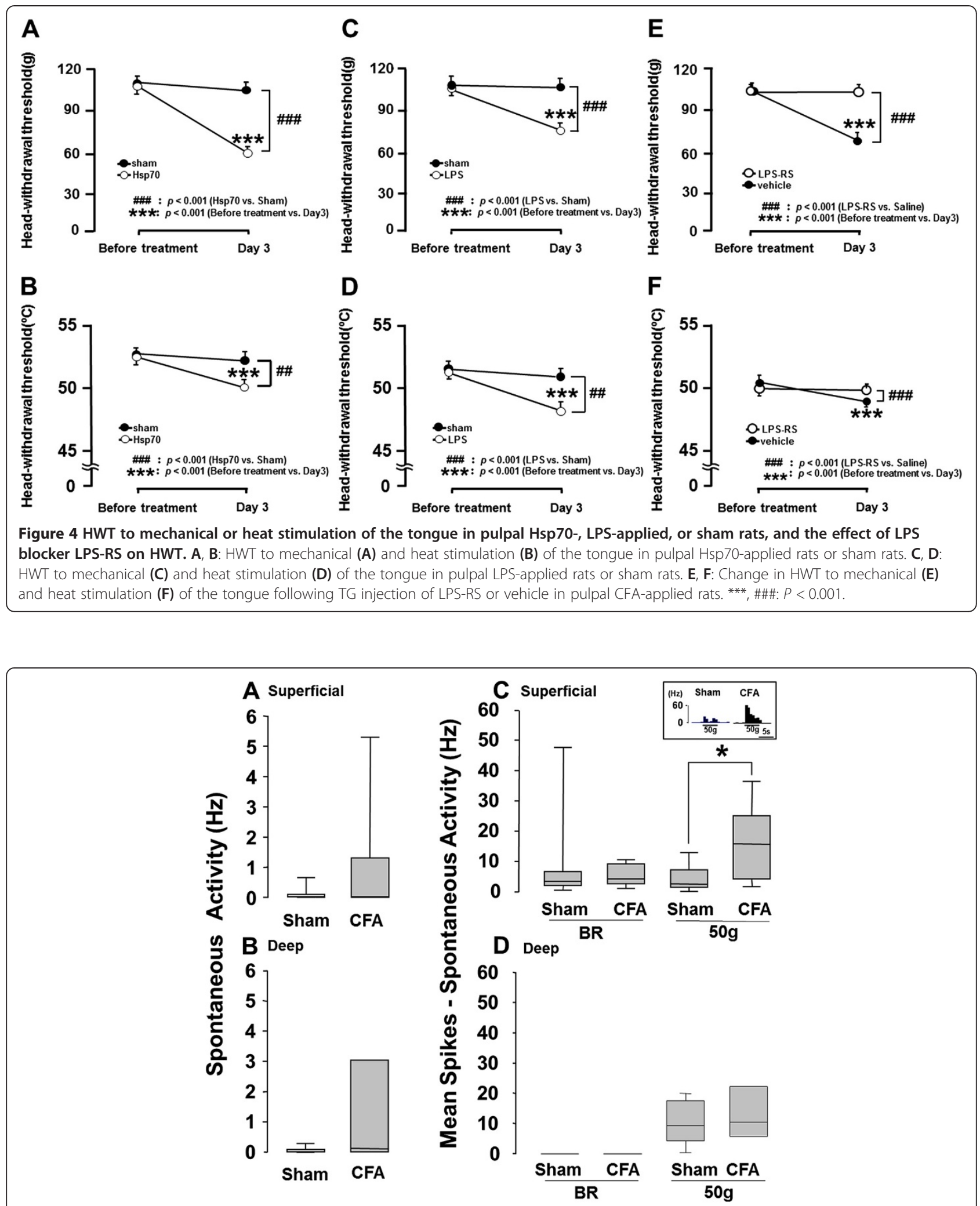

Figure 5 Spontaneous activities and brush or $50 \mathrm{~g}$ noxious mechanical responses of TG nociceptive neurons in M1TP CFA-applied rats or sham rats. A, B: Spontaneous activity of superficial RF neurons (A) and deep RF neurons (B), C, D: Mechanical evoked responses of superficial RF neurons (C) and deep RF neurons (D). Sham: M1TP sham-treated rats, inset diagram in $\mathbf{C}$ indicate typical unit activities of TG neurons, CFA: M1TP CFA-applied rats. *: $P<0.05$. 


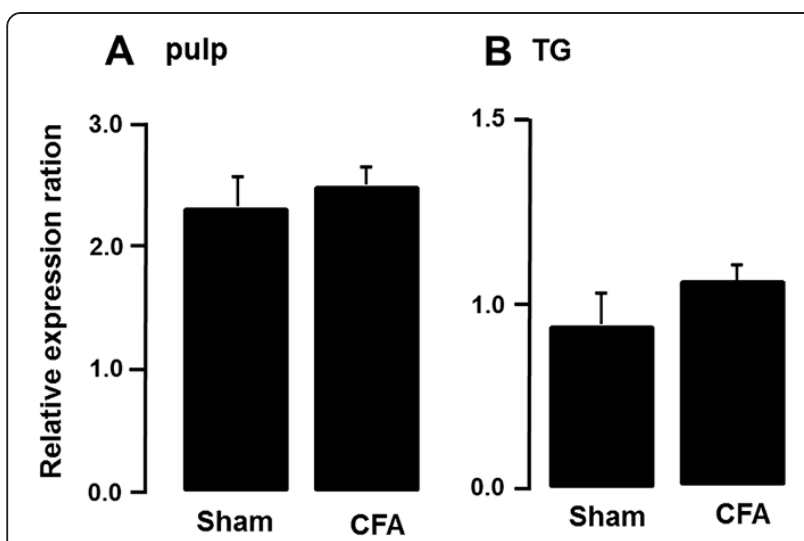

Figure 6 Relative change in Hsp70 mRNA levels in the M1TP or TG following CFA application to the M1TP or sham treatment of the M1TP. (A) M1TP; (B) TG.

Hsp70 in TG exerts immunomodulatory functions as TLR4 ligands to send peripheral neuroplastic changes or warning signals to the brain.

\section{Transportation of Hsp70 from M1TP to TG neurons}

It has been reported that Hsp70 is expressed in peripheral tissues as well as ganglion cells following tissue inflammation [25]. We hypothesized that Hsp70 was expressed in the M1TP associated with pulpal inflammation and transported from M1TP to TG neurons, or Hsp70 was expressed in TG neurons following pulpal inflammation. To evaluate if Hsp70 is transported to TG neurons following pulpal administration, Alexa-labeled Hsp70 protein was applied to the exposed M1TP in naive rats and analyzed the Alexa labeling in TG neurons. There were many Alexa-labeled neurons in the TG on day 3 after pulpal administration of Alexa-labeled Hsp70, and some of them were also labeled with FG injected into the tongue. We also did not observe differences of Hsp70 mRNA levels in the M1TP and TG between M1TP CFA-applied rats and sham-treated rats. It is likely that Hsp70 is expressed in the inflamed M1TP and transported to the TG neurons with axonal flow or Hsp70 is expressed in the TG neurons innervating in the tongue, and is released from TG neurons innervating the inflamed M1TP. Hsp70 released from TG neurons binds to TLR4, and the neuronal excitability of TG neurons innervating the non-inflamed tongue might be enhanced, resulting in the tongue ectopic pain associated with tooth pulp inflammation.

\section{TG neurons innervating tooth pulp and tongue}

We also observed that $9.2 \%$ of TG neurons were retrogradely labeled with FG and DiI injected into the M1TP and tongue, respectively. Our previous studies have reported that about $6 \%$ of TG neurons innervate multiple tooth pulps [15]. These suggest that some TG neurons innervate both M1TP and tongue, and this anatomical feature favors the development of ectopic tongue pain associated with M1TP inflammation.

\section{Conclusions}

These results are the first documentation that TLR4 is involved in the ectopic tongue pain associated with M1TP inflammation. Following M1TP inflammation, Hsp70 is expressed in the pulpal tissues, transported to the cell bodies of the trigeminal ganglion neurons, or Hsp70 is expressed in TG cells, and then released from TG neurons innervating the inflamed M1TP. Hsp70 binds to TLR4 in TG neurons innervating the tongue, and the excitability of TG neurons innervating the tongue may be enhanced, resulting in ectopic tongue pain.

\section{Abbreviations}

CFA: Complete freund's adjuvant; CNS: Central nervous system; DAMPs: Danger-associated molecular patterns; EMG: Electromyogram; FG: Fluorogold; Hsp70: Heat shock protein 70; HWT: Head-withdrawal reflex threshold; i.p.: Intraperitoneal; IR: Immunoreactive; LPS: Lipopolysaccharide; M1: Molar tooth pulp; NGS: Normal goat serum; PAMPS: Pathogen-associated molecular patterns; PNS: Peripheral nervous systems; TG: Trigeminal ganglion; TLR4: Toll-like receptor 4; TLRs: Toll-like receptors; TP: Tooth pulp.

\section{Competing interests}

All authors declare that they have no competing interests.

\section{Authors' contributions}

$\mathrm{KO}$ and $\mathrm{KS}$ participated in the design of the experiments, performed the animal studies, analyzed the data, and wrote the manuscript. SM executed the immunohistochemical analyses and revised the manuscript. BO participated in the design of the experiments and revised the manuscript. MA and DO performed the RT-PCR experiments. YT participated in the single neuron recording from TG neurons. MS and $\mathrm{KI}$ conceived and designed the study, analyzed the data, and wrote the manuscript. All authors read and approved the final version of the manuscript.

\section{Acknowledgements}

We thank Professor K. Ren for invaluable comments and correcting English usage on the manuscript. This study was supported in part by research grants from Sato and Uemura Funds from Nihon University School of Dentistry for MS (2013) and KS (2011), and a grant from Dental Research Center, Nihon University School of Dentistry; Nihon University multidisciplinary research grant and Individual Research Grant; a grant from the Ministry of Education, Culture, Sports, Science and Technology to promote multidisciplinary research Projects (KAKENHI (Grant-in-Aid for Young Scientist (B)) \#21791868 and \#23792192 to KS, \#22792021 to MS, KAKENHI

(Challenging Exploratory Research) \#24659832 to KI); grants from the Ministry of Education, Culture, Sports, Science, Technology to promote

multidisciplinary research projects 'Brain Mechanisms for Cognition, Memory and Behavior' and 'Translational Research Network on Orofacial Neurological Disorders' at Nihon University.

\section{Author details}

${ }^{1}$ Department of Endodontics, Nihon University School of Dentistry, 1-8-13 Kandasurugadai, Chiyoda-ku, Tokyo 101-8310, Japan. ${ }^{2}$ Divisions of Advanced Dental Treatment, Dental Research Center, Nihon University School of Dentistry, Tokyo 101-8310, Japan. ${ }^{3}$ Department of Pathology, Nihon University School of Dentistry, 1-8-13 Kandasurugadai, Chiyoda-ku, Tokyo 101-8310, Japan. ${ }^{4}$ Division of Immunology and Pathobiology, Dental Research Center, Nihon University School of Dentistry, Tokyo 101-8310, Japan. ${ }^{5}$ Department of Physiology, Nihon University School of Dentistry, 1-8-13 Kandasurugadai, Chiyoda-ku, Tokyo 101-8310, Japan. 'Division of Functional Morphology, Dental Research Center, Nihon University School of Dentistry, Tokyo 101-8310, Japan. ${ }^{7}$ Division of Applied System Neuroscience Advanced Medical Research Center, Nihon University Graduate School of Medical Science, 30-1 Ohyaguchi-Kamimachi Itabashi, Tokyo 173-8610, Japan. 
Received: 27 August 2013 Accepted: 14 November 2013

Published: 23 November 2013

\section{References}

1. Grushka M, Sessle BJ: Applicability of the McGill pain questionnaire to the differentiation of 'toothache' pain. Pain 1984, 19:49-57.

2. Ertekin C, Secil Y, Yuceyar N, Aydogdu I: Oropharyngeal dysphagia in polymyositis/dermatomyositis. Clinical Neurol Neurosurg 2004, 107:32-37.

3. Bender IB: Pulpal pain diagnosis-a review. J Endod 2000, 26:175-179.

4. Farella M, Michelotti A, Gargano A, Cimino R, Ramaglia L: Myofascial pain syndrome misdiagnosed as odontogenic pain: a case report. Cranio 2002, 20:307-311.

5. Ji RR: Peripheral and central mechanisms of inflammatory pain, with emphasis on MAP kinases. Current Drug Targets Inflamm Allergy 2004, 3:299-303.

6. Takeda M, Tanimoto T, Nasu M, Ikeda M, Kadoi J, Matsumoto S: Activation of NK1 receptor of trigeminal root ganglion via substance $P$ paracrine mechanism contributes to the mechanical allodynia in the temporomandibular joint inflammation in rats. Pain 2005, 116:375-385.

7. Okun E, Griffioen KJ, Mattson MP: Toll-like receptor signaling in neural plasticity and disease. Trends Neurosci 2011, 34:269-281.

8. Liu T, Gao YJ, Ji RR: Emerging role of Toll-like receptors in the control of pain and itch. Neurosci Bull 2012, 28:131-144.

9. Giffard RG, Han RQ, Emery JF, Duan M, Pittet JF: Regulation of apoptotic and inflammatory cell signaling in cerebral ischemia: the complex roles of heat shock protein 70. Anesthesiology 2008, 109:339-348.

10. Iguchi M, Littmann AE, Chang SH, Wester LA, Knipper JS, Shields RK: Heat stress and cardiovascular, hormonal, and heat shock proteins in humans. J Athletic Train 2012, 47:184-190.

11. Zhang Y, Wang YH, Zhang XH, Ge HY, Arendt-Nielsen L, Shao JM, Yue SW: Proteomic analysis of differential proteins related to the neuropathic pain and neuroprotection in the dorsal root ganglion following its chronic compression in rats. Exp Brain Res Exp Hirnforsc Exp Cerebr 2008, 189:199-209.

12. Pileggi $R$, Holland GR: The expression of heat shock protein 70 in the dental pulp following trauma. Dental Traumatol 2009, 25:426-428.

13. Zimmermann M: Ethical guidelines for investigations of experimental pain in conscious animals. Pain 1983, 16:109-110.

14. Katagiri A, Shinoda M, Honda K, Toyofuku A, Sessle BJ, Iwata K: Satellite glial cell $\mathrm{P} 2 \mathrm{Y} 12$ receptor in the trigeminal ganglion is involved in lingual neuropathic pain mechanisms in rats. Mol Pain 2012, 8:23

15. Omagari D, Takenouchi-Ohkubo N, Endo S, Ishigami T, Sawada A, Moro I, Asano M, Komiyama K: Nuclear factor kappa B plays a pivotal role in polyinosinic-polycytidylic acid-induced expression of human beta-defensin 2 in intestinal epithelial cells. Clinical Exp Immunol 2011, 165:85-93.

16. Diogenes A, Ferraz CC, Akopian AN, Henry MA, Hargreaves KM: LPS sensitizes TRPV1 via activation of TLR4 in trigeminal sensory neurons. J Dental Res 2011, 90:759-764.

17. Ferraz CC, Henry MA, Hargreaves KM, Diogenes A: Lipopolysaccharide from porphyromonas gingivalis sensitizes capsaicin-sensitive nociceptors. J Endod 2011, 37:45-48.

18. Qi J, Buzas K, Fan H, Cohen Jl, Wang K, Mont E, Klinman D, Oppenheim JJ, Howard OM: Painful pathways induced by TLR stimulation of dorsal root ganglion neurons. J Immunol 2011, 186:6417-6426.

19. Nicotra L, Loram LC, Watkins LR, Hutchinson MR: Toll-like receptors in chronic pain. Exp Neurol 2012, 234:316-329.

20. Hitomi S, Shinoda M, Suzuki I, Iwata K: Involvement of transient receptor potential vanilloid 1 in ectopic pain following inferior alveolar nerve transection in rats. Neurosci Letters 2012, 513:95-99.

21. Shinoda M, Asano M, Omagari D, Honda K, Hitomi S, Katagiri A, Iwata K: Nerve growth factor contribution via transient receptor potential vanilloid 1 to ectopic orofacial pain. J Neurosci 2011, 31:7145-7155.

22. Yasuda M, Shinoda M, Kiyomoto M, Honda K, Suzuki A, Tamagawa T, Kaji K, Kimoto S, Iwata K: P2X3 receptor mediates ectopic mechanical allodynia with inflamed lower lip in mice. Neurosci Letters 2012, 528:67-72.
23. Kim JY, Yenari MA: The immune modulating properties of the heat shock proteins after brain injury. Anatomy Cell Biol 2013, 46:1-7.

24. Guo LH, Schluesener HJ: The innate immunity of the central nervous system in chronic pain: the role of toll-like receptors. Cell Mol Life Sci: CMLS 2007, 64:1128-1136.

25. Turturici G, Sconzo G, Geraci F: Hsp70 and its molecular role in nervous system diseases. Biochem Res Int 2011, 2011:618127.

doi:10.1186/1742-2094-10-139

Cite this article as: Ohara et al:: Toll-like receptor 4 signaling in trigeminal ganglion neurons contributes tongue-referred pain associated with tooth pulp inflammation. Journal of Neuroinflammation 2013 10:139.

\section{Submit your next manuscript to BioMed Central and take full advantage of:}

- Convenient online submission

- Thorough peer review

- No space constraints or color figure charges

- Immediate publication on acceptance

- Inclusion in PubMed, CAS, Scopus and Google Scholar

- Research which is freely available for redistribution 